\title{
Report of the First Brazilian Symposium on Basic Research in HIV/ AIDS
}

\author{
Angra dos Reis, RJ, Brazil \\ September 13 - 16, 1995
Bernardo Galvão-Castro, Luiz Roberto Ribeiro Castelo Branco, Mariza Gonçalves Morgado*, Vera Bongertz*

\begin{abstract}
Laboratório Avançado de Saúde Pública, Centro de Pesquisa Gonçalo Moniz - FIOCRUZ, Salvador, BA, Brasil *Departamento de Imunologia, Instituto Oswaldo Cruz, Av. Brasil 4365, 21045-900 Rio de Janeiro, RJ, Brasil
\end{abstract}

During the first decade of AIDS epidemic in Brazil very little basic research on HIV-1 was performed in this country. In fact, the efforts of Brazilian scientists and the government policy were aimed to solve the immediate health problems created by the emergent HIV-1/AIDS epidemic. The First Brazilian Symposium on Basic Research in HIV/AIDS was held in Angra dos Reis, Rio de Janeiro, from 13 to 16 of September 1995, in order to disseminate scientific information and enhance national and international collaboration with a view of strengthening national research capabilities.

Opportunity was taken to posthumously honor Dr Marguerite (Peggy) Pereira and Dr Helio Gelli Pereira, two of the world's most distinguished virologists, whose life-work was presented by $\mathrm{Dr}$ Herman Schatzmayr (FIOCRUZ, Rio de Janeiro). These scientists contributed a great deal to solve some severe and important public health problems in Brazil. Thanks to their contributions it has been possible to achieve significant success in controlling the HIV/AIDS epidemic spread in blood donation centers in Brazil.

Dr Bernardo Galvão-Castro (FIOCRUZ, Salvador) summarized the main historical aspects of the AIDS epidemic in Brazil. He recalled that the first AIDS cases reported in Brazil date back from 1982, when six cases were reported in São Paulo and Rio de Janeiro. At that time, there was no official program to face this emergent epidemic. In fact, this program was established in 1985. Recognizing the severity of this new public health problem, some groups of Brazilian researchers, mainly in São Paulo and Rio de Janeiro, iniciated some activities in clinic, immunology and epidemiology of HIV/AIDS.

In July 1985 Drs Peggy and Helio Pereira kindly donated H9/HIV-1 infected cells to the Oswaldo Cruz Foundation. This enabled FIOCRUZ to promptly set up the HIV-1 screening routine in the public Brazilian blood bank network, using the immunofluorescence assay. The first isolation of
HIV-1 from a seropositive Brazilian individual was achieved in May 1987. During this time, a lot of effort was oriented towards solving emergent public health needs such as the development of easy, quick and cheap screening and confirmatory assays for the serological diagnosis. The importance of several institutions in financing reasearch projects, e.g. Fundação Banco do Brasil, National Research Council (CNPq) and Petrobrás was pointed out.

Representing the Ministry of Health, Dr Euclides A Castilho highlighted the trends of the HIV/AIDS epidemic in Brazil. He stated that in the beginning of the epidemic, the epidemiologic profile was similar to that observed in the USA and Western Europe. The homosexual/bisexual mode of transmission was the most important route in the propagation of infection in Brazil. Later, the epidemic profile began to change and currently the intravenous drug use and the heterosexual mode of transmission have assumed greater significance. He also summarized the present prevalence of HIV/AIDS in Brazil: as of February of 1995, a total of 71,111 AIDS cases were officially reported to the Ministry of Health. The infection is disseminated all over the country and the estimated prevalence of infection in terms of incidence per 100,000 inhabitants is 11.2 in the Northern, 13.0 in the Northeastern, 32.3 in the Middle-Western, 91.0 in the Southeastern and 26.5 in the Southern regions of Brazil. With respect to sexual preferences, since 1980 a total of $45 \%$ of the cases of HIV/AIDS occurred in homosexual/bisexual men and $17.7 \%$ in heterosexuals. The parenteral mode of transmission accounts for $25.2 \%$ of cases due to blood or blood factors and $21 \%$ due to intravenous drug use.

Dr Eduardo Martins, Vice-President of the FIOCRUZ, drew attention to the fact that the Oswaldo Cruz Foundation, as the main research institution of the Ministry of Health, recognizes the urgent need to establish ways to face the HIV-1 epidemic in Brazil as well as other emergent or reemergent infections. Therefore, the Institutional 
Program of AIDS was set up as a pilot program in 1991, in order to obtain more skill, knowledge and efficacy in the control of this devastating epidemic. The program involves multidisciplinar activities in the areas of basic, clinical, epidemiological and educational research.

Dr Herbert de Souza, President of the Brazilian Interdisciplinary AIDS Society (ABIA) called attention to a very important aspect of the HIV-1 epidemic in Brazil: the dissemination to the lowincome population. This fact will have very important implications in the overall control of HIV/ AIDS in Brazil. Taking this in consideration, he stated that it is extremely urgent to strengthen and amplify the public health services. He also emphasized the importance of the integration of basic research with clinical and social activities to control this infection. Finally, he reaffirmed that the society has great confidence, expectations and hope in the joint efforts of the scientists to surmount this epidemic.

\section{ANTI-HIV/AIDS VACCINES}

A review on the actual stage of development and evaluation of anti-HIV/AIDS vaccine candidates worldwide was presented by Dr S Osmanov of the Vaccine Development Unit of the Global Programme on AIDS of the World Health Organization. Relevant information presented included the tendency of the transformation from an AIDS epidemy to a worldwide disease, with a frightening increase of actual and predicted infection rates for mainly Asia and Africa but also for Latin America. The extreme genotypic variety of HIV in different geographical areas was discussed, as well as the shift in predominant genotypes. The necessity of the establishment of a correlation between genotypic characteristics of the viral variants with the immune response of the infected individuals (serotyping, analysis of functional HIV neutralizing antibodies) for choice of vaccine candidates in different geographical areas, as well as the study of the importance of factors affecting natural resistance and long-term non-progression was pointed out. A rapid review of anti-HIV/AIDS vaccine candidates was presented, stressing the promising results obtained with the use of live vectors followed by booster immunizations with proteins or peptides. The need for more detailed studies aiming to answer critical questions still to be solved, such as the necessity of obtaining heterotypic immune responses, the importance of inducing not only systemic but local protection, most importantly at mucosal levels, the lack of data concerning the influence of secondary and opportunistic infections, such as other sexually transmitted diseases, the need to protect not only adults but also children against HIV infection or at least the associated disease, among questions related to risk/benefit analyses which have to be considered before entering into studies related to the vaccination strategies (phase IV), predicted to start as late as 2005 .

The results obtained by the WHO Network for HIV isolation and characterization, established in order to answer some of the questions presented above, were outlined by Dr J Mullins (University of Washington, Seattle). The study aimed to analyse the variability of HIV in different geographical regions and to establish the importance of HIV variation at the level of the hosts immune response induced. Results obtained up to now indicate the low correlation between genotypic and serotypic analyses and the lack of correlation between genotypes and the kinds of HIV neutralizing antibodies induced. However, interesting new data has been established by phenetic analysis (B Korber), indicating that although the V3 loop of the gp120 envelope glycoprotein cannot be defined as the linear "principal neutralization domain" any more, the conformation of the V3 loop, as affected by charged amino acids, does correlate with the biological characteristics of the HIV variant in question. As positive correlation between biological characteristics (cytopathogenic effect) and disease progression has been established, the data indicating that conformation of the V3 loop (as predicted by genetic analysis) can be associated to prognosis of HIV infection.

Dr CV Hanson (CA Dept Health Services, Bethesda) presented data on the HIVNET established in the USA for evaluation of anti-HIV/AIDS vaccine studies, indicating the protocol for phase I/II trials followed in the USA, stressing the high number of volunteers needed for meaningful analyses in low incidence areas. The particular efforts of the CA Dept of Health Services on analysis of the HIV neutralizing antibody response of vaccinated individuals, using different assays and quantifying analyses were presented, including the preliminary results obtained which indicate a lack of widerange neutralizing antibody response against different HIV variants detected in the vaccinees.

An interesting pre-clinical trial was described by JG Guillet (I Pasteur, Paris), in which specific cytotoxic $\mathrm{T}$ lymphocytes could be induced by immunization of mice and of macacques with HIV-1 peptides (env, nef, gag) coupled to a lipidic tail. A trial in human volunteers is being initiated.

\section{GENE THERAPY}

A review of recent progress in gene therapy anti-HIV/AIDS was presented (O Ferreira, UCLA, Los Angeles), discussing strategies employing 
HIV-1 specific ribozymes, antisense RNA, RNA decoys and expression of mutant HIV genes with dominant repressor activity. Particularly experiments related to this last approach, using the necessity of HIV-1 for the human t-RNA/Lys3 for reverse transcription, were presented. Results of in vitro experiments indicate that susceptibility to HIV-1 infection can be reverted by transduction of a lymphocytic cell line with a mutant tRNA (tRNAtarD).

Dr F De Lucca (USP, Ribeirão Preto) presented interesting results about the in vitro transfer of cellular immunity to human lymphocytes with exogenous RNA obtained from mice immunized with synthetic peptides corresponding to immunologically important HIV-1 epitopes, and discussed the potential use of this methodology for immunotherapy.

\section{MUCOSAL IMMUNITY}

Prof GE Griffin (University of London) described how HIV infects mucosal tissues. He showed results of in vitro studies of infection of human mucosal tissue - gut and cervix explants by HIV, demonstrating that gut and cervical immune cells are potential targets for direct HIV infection. His studies on nutrition have demonstrated that the majority of patients within the initial phases of AIDS can gain weight and that weight loss is associated with lower food intake and not with energy loss as hypothesized.

The human gastrointestinal immune responses to HIV was discussed and results of studies done in Europe and Africa were shown, which suggest that the mucosal system is able to respond well to oral immunogens until relatively late in HIV disease (LRR Castello-Branco, FIOCRUZ/Banco do Brasil, Rio de Janeiro).

\section{IMMUNOPATHOLOGY}

The alterations found in lymph nodes of HIV-1 positive patients were described (D Paiva, UERJ, Rio de Janeiro). Her studies, using histological methods and immunocytochemistry, showed that during progression of HIV disease to AIDS different patterns of abnormalities were seen, from an initial follicular hyperplasia to a subsequent follicular involution.

\section{HIV NEUTRALIZATION}

The importance of HIV neutralizing antibodies in pathogenesis, transmission and protection studies was discussed by Dr EM Fenyö (Karolinska Institute, Stockholm). Recent data indicating the positive correlation between neutralizing antibodies and slower disease progression was established by analysis of primary HIV infection, analysing sequential HIV isolates. Studies of vertical HIV transmission established significant correlation between range of HIV neutralization and lower risk of HIV transmission from mother to child (G Scarlatti et al.). Similar results were found in a study of heterosexual HIV transmission. Attempts to associate genetic subtypes with "neutralization serotypes" have not been successful, although neutralization subtypes appear to exist.

Description of the cryptic nature of envelope gp120 V3 loop neutralizing epitopes in primary monocytotropic HIV-1 was given by Dr DC BouHabib (FDA, NIH, Bethesda). Studies carried out using a novel CD4+ T-cell line (PM-1) permissive for both monocytotropic and T-cell-tropic virus types permitted a comparative analysis of neutralization of two viral variants with rabbit antiserum to a recombinant of the monocytotropic variant gp120 and anti-V3 monoclonal antibodies. The resistance of the primary monocytotropic variant to these antibodies contrasted with the susceptibility of the T-cell-tropic variant maintained in T-cell line cultures.

Results on studies of neutralizing antibodies in Brazil were presented, indicating that both in São Paulo (LFM Brígido, Adolfo Lutz Institute, São Paulo) and in Rio de Janeiro (V Bongertz, FIOCRUZ, Rio de Janeiro) the presence of neutralizing antibodies active against autologous and heterologous HIV-1 variants isolated from individuals residing in Brazil could be detected, with results comparable to those obtained in other parts of the world. Data indicating a reverse correlation between 50\% neutralization susceptibility of Brazilian primary HIV-1 isolates and lack of potency of the corresponding antisera in neutralizing heterologous primary isolates were presented.

\section{CYTOTOXIC T LYMPHOCYTES}

Protective response against HIV has been correlated to neutralizing antibodies and cytotoxic $\mathrm{T}$ lymphocytes (CTL), therefore these immune responses are attempted to be induced by anti-HIV/ AIDS vaccine candidates. The difficulties in inducing autologous neutralizing antibodies, the decreasing response of autologous neutralizing antibodies with progression of disease and data indicating a loss of Th1 response with disease progression have focused interest in the cellular response against HIV-1. Studies with peptides derived from env, gag and nef regions of HIV-1 have indicated not only induction of specific CTL but also a positive correlation between CTL response and reduction of viral load as well as a slowing down of disease progression (JG Guillet).

The high frequency detection of CD45RO precursor CTL for HIV-1 Nef protein in unexposed 
human blood donors was presented by Dr MA Lucchiari-Hartz (Butantan Institute, São Paulo). The generation and characterization of Nef-specific cytotoxic T cell responses of most tested non-infected subjects through in vitro stimulation with autologous EBV-transformed lymphoblastoid B cell lines expressing Nef-protein was described. These CTLs were characterised as CD3+CD4- CD8+ and MHC class I restricted cells. Both CD45RO and CD45RA were able to generate Nef-specific and EBV-specific CTL responses, with a higher frequency in the CD45RO subset.

\section{HIV-1 POLYMORPHISM}

A study of the molecular variability of HIV-1 and its implication on cell tropism and pathogenicity was presented by Dr C Cheng-Mayer (Aaron Diamond AIDS Research Center, New York). The importance of the stage of the disease for virus isolation and characterization, of the amino acid sequence, charge and quaternary conformation of the envelope gp120 V3 loop in phenotypic characteristics of the HIV-1 isolate was discussed. Results of comparative studies of the T-cell-line adapted, syncytium inducing HIV-1 SF2 isolate and the monocytotropic, non-syncytium inducing HIV1 isolate SF162 were presented, comparing the V3 loop amino acid sequences and their susceptibility to neutralizing monoclonal antibodies and to the inhibitory effect of soluble CD4. The importance of studying biological characteristics of HIV-1 isolates with polymeric envelope gp120 in contrast to monomeric recombinant gp120 analyses was pointed out.

An extensive evaluation of HIV diversity in Brazil was presented in this meeting by the group of Brazilian researchers participating in the $\mathrm{Na}-$ tional Network on HIV Isolation and Characterization. Using the heteroduplex mobility assay to define HIV-1 subtypes of isolated viruses or of peripheral mononuclear cells from HIV-1infected individuals. Dr E Sabino (Adolpho Lutz Institute, São Paulo) showed the presence of the B and F subtypes among intravenous drug users in São Paulo, suggesting that the frequency of F subtype is higher in this group. Using the same methodology, Dr PC Ferreira (UFMG) evaluated 18 samples obtained from blood donors and hemophiliac patients in Belo Horizonte, MG. While all hemophiliac patients (8) were infected with subtype B, 2 out of the 10 blood donors analyzed were infected with subtype F. Similarly, Dr MG Morgado (FIOCRUZ, Rio de Janeiro) also showed the presence of the B and F HIV-1 subtypes in Rio the Janeiro. Although her data are suggestive of an increase of the frequency of the F subtype, no association between subtype distribution and sex or risk group could be established. Results were also presented of the lymphoproliferative response of HIV-1 infected individuals to 15 mer synthetic peptides corresponding to the crown of the V3 loop . Despite inter-isolate peptide sequence heterogeneity, HIV-1 positive individuals reactive to the typical Brazilian HIV-1 B peptide (GWGR) had broad lymphoproliferative reaction with other peptides such as the MN, LAI, SF-2 or B consensus (GPGR), suggesting conservative T cell epitopes in the region covered by those peptides (MG Morgado, FIOCRUZ, Rio de Janeiro). Using another approach, based on the restriction fragment length polymorphism of the PCR amplified protease gene, Dr A Tanure (UFRJ, Rio de Janeiro) showed dual infections with distinct HIV-1 subtypes in HIV positive individual from Rio de Janeiro. Indeed, infection with HIV-1 B and F subtypes was detected in one individual, with subtypes $\mathrm{F}$ and $\mathrm{D}$ in another and with subtypes $\mathrm{C}$ and $\mathrm{D}$ in a couple and, possibly, their child. These data suggest that infection with one HIV-1 subtype does not protect against superinfection with other subtypes.

One of the points discussed was the effort to establish serotypes of HIV-1 and the attempts to correlate serotypes and genotypes. The data obtained by the WHO Network for HIV Isolation and Characterization indicate that there is no absolute correlation between genotypes and serotypes of HIV-1, although it was possible to separate A/C genotypes as one serotype (94\%), genotype E as another serotype (93\%) and B and B' as a distinct serotype (80\%), although relatively extensive cross-reactivity could be established between genotypes (J Mullins). An assay able to differentiate between the North-American/European B genotype and the B' genotype detected in Brazil was presented, employing biotinilated peptides, indicating that the specific affinity of anti-B' antibodies to B' peptides could be used for a positive serotyping assay (CV Hanson).

\section{DISEASE PROGRESSION MARKERS AND DIAGNOSIS}

Dr RS Pereira (University of London) discussed some results from studies carried out in England on immunological markers for HIV progression. He pointed out that CD4 T cell count, measured by flow cytometry, is still the best investigation test. As this test is expensive, a suggestion was made that clinicians should use this tool to follow decrease of CD4 count, avoiding tests when CD4 count is below $50 / \mu \mathrm{l}$ as the number seldomly increases thereafter.

The cellular immune responses in HIV infected subjects and the mechanisms that cause depletion 
of CD4+ cells during HIV infection were discussed by Dr A Duarte (USP, São Paulo). He pointed out that while $\mathrm{CD} 4 \mathrm{~T}$ cells decrease in number, the expression of CD4 molecules on the surface of these cells increases. He presented results on immunological markers during infection showing increase of $\mathrm{IgG}$ in serum, decrease of delayed-type hypersensitivity and dysfunction of helper $\mathrm{T}$ cell responses.

The hypothesis that there is a switch from the Th1 cytokine profile to Th0/Th2 during evolution to AIDS was discussed (LS Camargo, USP, São Paulo). This switch is important in inducing eosinophilia, increase of serum IgE and IgA, CD4 $T$ cell decrease, increase of side effects to drugs and decrease of delayed-type hypersensitivity.

A new diagnostic PCR HIV test to be used on dried blood spots on filter paper was presented (S Cassol, British Columbian Center for Excellence in HIV/AIDS, Vancouver). This test presents high sensitivity (94.7\%) and specificity (100\%). When used in newborn children, the sensitivity on days 0 to 4 is $27 \%$, increasing on days 10 to $15(89 \%)$ and reaching its maximum (100\%) from day 16 onwards.

The usefulness of detection of anti-reverse transcriptase antibodies (RTI) in HIV-1 infection as a progression marker was discussed by $\mathrm{Dr} \mathrm{M}$ Ueda (Adolfo Lutz Institute, São Paulo). Results on detection of RTI in 80 to $90 \%(n=20)$ asymptomatic HIV-1 infected individuals and sequential followup of RTI titers indicated a significant decrease in RTI titers in correlation to disease progression. Correlation of the decrease in RTI titers with increase in beta-2-microglobulin detection in individuals with fast disease progression was statistically significant. A study of 54 asymptomatic males (homosexual transmission of HIV-1) indicated an inverse correlation between viral load and RTI titers.

Results of analyses of children of HIV-1 infected mothers obtained with antibody detection, p24 determination, HIV-1 isolation and PCR detection of proviral DNA in 44 children were presented (JP Simonetti, FIOCRUZ, Rio de Janeiro).

\section{CO-INFECTIONS}

One of the most important aspects discussed in this Symposium concerned the immunopathological features of co-infections between HIV-1 and other infectious diseases endemic in Brazil.

In this context, tuberculosis represents one of the most frequent diseases associated to HIV infection in Brazil and its diagnosis, depending on the patient, can be predictive of HIV infection. In this meeting, John Ho (Cornell University, New York) discussed the hyphotesis that co-infections accentuate the intrinsic immunopathology of HIV infection, shortening the HIV disease free interval and survival. Moreover, he also provided evidence that co-infections with sexually transmitted diseases can enhance HIV replication and transmission. Co-infections might enhance HIV replication by direct or indirect mechanisms or, alternativelly, co-infections might result in immune activation, suppression or immune cell depletion that would affect HIV replication or contribute to immunessuppression.

The immunopathological aspects of the association between HIV infection and tuberculosis were studied in a phenotyping analysis of cell populations from the bronchoalveolar lavage and from the peripheral blood obtained from AIDS patients and those co-infected with Mtb, as well as their functional activity determined (MG BoneciniAlmeida, FIOCRUZ, Rio de Janeiro/Cornell University, New York). Low lymphoproliferative response to PPD and Mtb antigens was verified for the cells from the bronchoalveolar lavage in the co-infected HIV/Mtb individuals, in addition to the reduced phagocytic activity of monocytes/macrophages and neutrophils. She also showed the expression of inducible nitric oxide synthase in human macrophages as well as its envolvment in the anti- Mtb activity of human macrophages.

In another attempt to understand the mechanisms envolved in the hosts defense against Mtb, Dr JR Lapa e Silva (FIOCRUZ-UFRJ, Rio de Janeiro) evaluated the phenotype of the mononuclear cells obtained from lungs of patients with active tuberculosis. Increased expression of HLA$\mathrm{DR}$, a marker of celll activation, in addition to a significantly higher proportion of cells expressing dendritic and epitelioid cell markers was verifyed in the tuberculosis group when compared to normal controls, suggesting that the recruitment of monocytes and local cell activation are not sufficient to control this pathogen.

American tegumentary leishmaniais (ATL) is another disease endemic in Brazil. Its association with HIV infection has been published by different groups. As shown by Dr SG Coutinho (FIOCRUZ, Rio de Janeiro), lesions and high parasite load were associated to a clear depression of $\mathrm{T}$-cell mediated immune response to Leishmania antigens. The majority of the responsive cells to Leishmania antigens had the CD8+ phenotype, suggesting the involvement of this $\mathrm{T}$ cell population and the IFN-g production in the cure of localized cutaneous leismaniasis.

Another relevant aspect discussed in this meeting was the association between HIV and $\mathrm{Myco}-$ bacterium leprae infections. As discussed by $\mathrm{Dr} \mathrm{E}$ Sarno (FIOCRUZ, Rio de Janeiro), despite of the 
low CD4+ counts in peripheral blood, no histopathological or phenotypic alterations were observed in the lesions of lepromatous and tuberculoid HIV positive patients, when compared to HIV negative individuals. The reactivity to the Mitsuda test as well the the lymphoproliferative response to $M$. leprae antigens, usually detected in patiens with tuberculoid lepra, was abrogated in the HIV-1 co-infected individuals. However, the IFN-g production in response to the same antigens was similar in both groups. In addition, overexpression of cytokine genes in non-stimulated peripheral mononuclear cells from co-infected individuals was verified, giving evidence for cytokine gene expression even in very low CD4+ cell counts.

Cerebral toxoplasmosis is one of the most important opportunistic diseases associated to HIV infection in Brazil, placed in the fourth position in the Epidemiological Report (PN-DST/AIDS, Brazilian Ministry of Health, Jun-Aug, 1995). Based on a in vitro model of HIV-1/Toxoplasma gondii co-infection, Dr R Gazzineli (UFMG/FIOCRUZ, Belo Horizonte) showed that in monocyte/macrophage cell cultures $T$. gondii activates and/or potentiates HIV-1 replication, possibly by the induction of monocyne production. The potential importance of this enhancement of the HIV replication on the evolution to AIDS was also discussed.

Concerning the Brazilian National Plan of HIV1/AIDS vaccine evaluation, Dr Euclides A Castilho (FIOCRUZ, Rio de Janeiro/Ministry of Health,DF) informed that in 1991 Brazil has been selected by the Global Programme on AIDS of the World Health Organization as a site for potential vaccine efficacy trials. This plan contemplates ethical aspects mainly related with respect, benevolvence, non-malevolence and justice and involves four different areas, namely virology, clinical studies, epidemiological studies and sexual behaviour.

Currently, three vaccine sites have been established in Brazil: Belo Horizonte, MG, Rio de Janeiro, RJ and São Paulo, SP. These three centers have conducted research projects with the following objectives: (a) to evaluate the feasibility of establishing and maintaining male homosexual/bisexual cohorts, (b) to determine soro-incidence of HIV infection, (c) to conduct social behavioral studies in order to understand better the different risk factors for HIV infection.

Dr Dirceu Greco (UFMG, Belo Horizonte) reported briefly on the first phase I trial in Brazil. The HIV-1 synthetic peptide product used in this trial was produced by the United Biomedical Inc., USA. This product is composed of an octameric peptide linked by heptalysin, corresponding to the V3 loop of HIV-1 MN isolate. As prescribed in the National Plan for HIV-1 Vaccine Evaluation, this vaccine had been previously tested in the USA. The general objective of the trial is to evaluate immunogenicity and safety of this product in our human and environmental conditions with randomized, double-blinded and controlled studies. These studies are underway in Belo Horizonte as well as in Rio de Janeiro and include 15 volunteers in each state.

Based on HIV-1 diversity observed in Brazil, Dr Bernardo Galvão-Castro briefly summarized the Ministry of Health strategy to systematically monitor HIV-1 polymorphism in Brazil. For this purpose, a National Laboratory Network fot HIV-1 Isolation and Characterization was established. The main objectives of this network are: (1) to develop a system for monitoring the antigenic variability of HIV-1 isolates from different regions of Brazil, (2) to generate basic information of genetic and antigenic properties of epidemiologically relevant HIV-1 strains that will enable the selection of antigenically appropriate candidate vaccines to be evaluated and potentially used in Brazil, (3) to participate in other international HIV-1 characterization efforts as a part of the WHO Network for HIV Isolation and Characterization, on a bilateral basis of collaboration with individual international research programs.

The necessity of maintaining contact between individual research groups in Brazil, in their national and international research efforts in the field of basic research revealed in this first Symposium was discussed. It was decided to hold bienal small meetings such as this first Symposium, and the FIOCRUZ confirmed its inclination to support future meetings.

This is a Personal View of the First Brazilian Symposium on Basic Research in HIV-1/AIDS, with special emphasis on results presented but not published in this journal issue, as expressed by the authors. 